\title{
Mindfulness y cáncer: aplicación del programa MBPM de respira vida breathworks en pacientes oncológicos
}

\author{
Lorena Alonso Llácer 1,*, Marta Ramos-Campos ${ }^{2}$ \\ 1 Psicooncóloga en Junta Provincial asociada de Valencia de Asociación Española Contra el Cáncer, Doctora \\ en Psicología. \\ 2 Psicóloga en Junta Provincial asociada de Valencia de Asociación Española Contra el Cáncer, Máster en \\ Psicología General sanitaria. \\ * Autor correspondencia: turmalina29@hotmail.com
}

Recibido: 31/10/2018; Aceptado: 21/11/2018; Publicado: 30/11/2018

Resumen: El cáncer es una enfermedad frecuente y compleja que compromete la supervivencia de la persona que la padece, y genera un estrés socioemocional que condiciona la calidad de vida y justifica la demanda de atención psicoterapéutica. Estudios previos muestran que un programa basado en mindfulness contribuye a un mayor ajuste psicológico en los pacientes oncológicos, reducción de los niveles de ansiedad y de depresión, mejoría de la calidad del sueño, uso de mejores estrategias de afrontamiento, cambios en el sistema inmune y endocrino, aumento de la calidad de vida y reducción del dolor. El objetivo del presente estudio es valorar la mejora del ajuste psicológico del paciente oncológico a través de la aplicación del programa de tratamiento MBPM. La muestra de pacientes $(\mathrm{N}=22)$ fue derivada de la unidad de psicooncología de la Asociación Española Contra el Cáncer de Valencia. Todos los sujetos fueron entrevistados y evaluados antes de la intervención y después de la misma, mediante una batería de cuestionarios. El programa MBPM creado por Vidyamala Burch. Se trata de una terapia grupal consta de 8 sesiones de 2,5 horas de duración y frecuencia semanal, que enseña una forma más sana de relacionarse con el sufrimiento, mediante la autogestión. Tras la intervención se observa una mejoría significativa en la escala que mide capacidad mindful, en la de autocompasión y en el bienestar psicológico, satisfacción con la vida y vitalidad subjetiva y una reducción significativa del dolor $(\mathrm{p}<0.05)$. Tal y como muestran los resultados, mejora el ajuste psicológico de los pacientes. Cabe destacar la importancia de replicar este estudio con un grupo control y un $\mathrm{N}$ mayor para poder generalizar estos resultados, aunque los resultados son prometedores.

Palabras Clave: Mindfulness; Compasión; Dolor; Bienestar psicológico; Cáncer; Regulación emocional; Autocompasión.

Abstract: Cancer is a frequent and complex disease that compromises the survival of the person
who suffers it, and generates a social-emotional stress that conditions the quality of life and justifies
the demand for psychotherapeutic care. Previous studies show that a program based on
mindfulness contributes to a greater psychological adjustment in oncological patients, reduction in
levels of anxiety and depression, improvement of sleep quality, use of better coping strategies,
changes in the immune system and endocrine, increased quality of life and pain reduction. The aim
of the present study is to assess the improvement of the psychological adjustment of the oncological
patient through the application of the MBPM treatment program. The sample of patients (N = 22)
was derived from the psycho-oncology unit of the AECC Valencia. All the subjects were interviewed
and evaluated before and after the intervention, through a battery of questionnaires. The MBPM
program created by Vidyamala Burch. It is a group therapy consists of 8 sessions of 2.5 hours and
weekly frequency, which teaches a healthier way of relating to suffering, through self-management.
After the intervention a significant improvement was observed in the scale that measures mindful 
ability, in the self-compassion and in the well-being psychological, satisfaction with life and subjective vitality and a reduction of pain $(p<0.05)$. As the results show, it improves the psychological adjustment of the patients. It is important to highlight the importance of replicating this study with a control group and a higher $\mathrm{N}$ in order to generalize these results, although the results are promising.

Key words: Mindfulness; Compassion; Pain; Well-being; Cancer; Emotional-regulation; selfcompassion;

\section{Introducción}

El cáncer es una enfermedad invalidante que genera un gran impacto psicológico, tanto en las personas afectadas como en sus familiares y personas cercanas [1,2]. Es una enfermedad crónica que cursa con un gran estrés desde su diagnóstico y a lo largo de todo el proceso. Estudios al respecto han mostrado que las personas con este tipo de diagnóstico presentan altos niveles de ansiedad y depresión [3,4].

El enfermo oncológico ha de adaptarse a una nueva situación que conlleva numerosas alteraciones emocionales y funcionales en su vida diaria. La problemática más frecuente suele ser: altos niveles de sufrimiento, malestar psicológico, aparición de miedos, deterioro de las relaciones sociales, estados de ánimo depresivos, ansiedad, problemas referentes a la sexualidad, cambios en la imagen corporal y menor autoestima [3,5-10].

En vista del sufrimiento por el que pasan tanto pacientes como familiares a lo largo del proceso de la enfermedad, son altamente recomendables las intervenciones psicológicas, pues ayudan a generar un afrontamiento activo y adaptativo por parte del paciente, repercutiendo en la mejora de su bienestar psicológico y calidad de vida [6,7].2. Material y Métodos

Esta sección se debe dividir en sub-apartados. Los materiales y métodos deben describirse con suficiente información para permitir a otros a reproducir y basarse en los resultados publicados. Tenga en cuenta que la publicación de su manuscrito implica que usted debe describir con detalle todos los materiales, datos y protocolos asociados con la publicación. Protocolos y los nuevos métodos deben describirse en detalle mientras que métodos bien establecidos pueden ser brevemente descritos y debidamente citados.

\section{Mindfulness en oncología}

Mindfulness se define como "atención plena", y se considera una terapia de tercera generación que ahonda sus raíces en la filosofía oriental. Los tres elementos esenciales que definen mindfulness son la aceptación, no juzgar y la vivencia del momento presente [11].

La conciencia plena es un estado mental en el que el individuo es altamente consciente y enfoca la realidad del momento presente, con apertura y sin juzgar ni dejarse llevar por pensamientos o reacciones emotivas. Por tanto, requiere una intención especial que reduzca al mínimo la utilización del "piloto automático", manteniendo la conciencia en lo que ocurre "aquí y ahora" [12].

Según Thich [13], mindfulness puede definirse como "mantener la consciencia habitando la realidad presente". Según Lutz, Dunne y Davidson [14] es un "estado en el cuál el practicante es capaz de mantener la atención centrada en un objeto durante un tiempo ilimitado. Ambas definiciones tienen en común esa capacidad de centrar la atención en el presente inmediato, que precisamente es la instrucción básica en mindfulness. Pero sólo con la instrucción ya mencionada no es suficiente, pues se requiere una actitud de aceptación, de apertura, curiosidad, paciencia, constancia y amor.

Una de las razones por las que el uso de la atención plena está teniendo éxito es porque ayuda a restablecer el equilibrio emocional y favorece la presencia de estados de ánimo positivos y las actitudes de aproximación frente a las de evitación, razón por la que se ha utilizado en el tratamiento de la depresión $[15,16]$.

Brown y Ryan [17] han argumentado que mindfulness facilita el bienestar psicológico de una forma directa, ya que aporta claridad y viveza en las experiencias a través de una apertura de los 
sentidos, sin el denso filtro de los pensamientos que distorsionan la realidad [18,19]. Por otro lado, mindfulness aporta bienestar psicológico al operar de forma indirecta a través del funcionamiento de la regulación del self, a través de una atención sensible a todos los aspectos psicológicos, somáticos y claves ambientales [18].

La práctica de mindfulness es tan eficaz para aliviar la ansiedad, el estrés y la depresión como el counselling y la medicación, como muchas investigaciones han demostrado. Estudios previos muestran que reduce el dolor con la misma eficacia que los calmantes y la morfina [20]. La meditación provoca cambios cerebrales, ya que amortigua las pautas cerebrales asociadas al dolor, traduciéndose estos cambios con el tiempo en una menor percepción del dolor. La mente tiene la capacidad de controlar el volumen que regula tanto la intensidad del dolor, como su duración. Cuando la mente entra en las espirales negativas a través del miedo, la tensión, los pensamientos encadenados, los recuerdos de experiencias de sufrimiento, se van superponiendo y van aumentando el volumen, predisponiéndonos a sufrir (sufrimiento secundario). En la práctica, uno puede tener dolor, pero no implica que tenga que sufrir. La práctica de mindfulness ayuda a cerrar las compuertas del dolor a través de la disolución del sufrimiento secundario [21].

Teniendo en cuenta la literatura científica, mindfulness se está recomendando para infinidad de enfermedades como el cáncer (efectos secundarios de la quimioterapia), enfermedades cardíacas, artritis, diabetes, fibromialgia, migrañas, enfermedades autoinmunes (esclerosis múltiple y el lupus), síndrome de fatiga crónica, colon irritable, dolor de espalda, etc. [21].

La práctica de mindfulness permite llegar a un estado de ecuanimidad donde el recipiente de la conciencia se amplía integrando tanto lo desagradable como lo agradable. De esta forma las personas son capaces de distanciarse de su sufrimiento, pues al convertirse en observadores de sí mismos, dejan de etiquetarse a sí mismos como enfermos. La práctica les permite desarrollar sensibilidad para escuchar su cuerpo y sus necesidades, en vez de reaccionar con el piloto automático, y así poder responder de la forma más sabia y creativa [12]. Sin duda esa forma distinta de relacionarse con el dolor y la enfermedad contribuye al bienestar emocional, desde una perspectiva holística e integrativa del ser humano.

Tacón [22] realizó una investigación para evaluar en qué medida la aplicación de un programa basado en mindfulness contribuía en mujeres diagnosticadas de cáncer de mama a aumentar su bienestar espiritual, por un lado, y por otro reducir los indicadores de pérdidas (independencia, funciones físicas y psicológicas) y los síntomas de duelo anticipado por su propia muerte. Los hallazgos encontrados en dicho estudio van en la línea de la hipótesis formulada por los investigadores (aumento del bienestar y reducción de los indicadores de pérdida y los síntomas de duelo anticipado).

Por otro lado, en el estudio realizado por León et al. [23] se comparó la efectividad de una intervención de MBSR (Reducción del estrés basada en mindfulness) con una intervención psicoeducativa estándar en pacientes oncológicos. En el grupo experimental (MBSR) se hallaron diferencias significativas indicativas de mejoría en todas las medidas (depresión, ansiedad-estado, ansiedad-rasgo y calidad de vida) mientras que en el grupo control sólo aparecieron en la variable ansiedad-estado. Los datos de esta investigación indican que el MBSR produce una mejoría importante en el malestar emocional y en la calidad de vida, superior a la que pueda lograrse con una intervención psicoeducativa. No obstante, no puede afirmarse que el MBSR deba ser la técnica de elección para todos los casos, pues no está claro que pueda ser seguida por cualquier tipo de paciente, al requerir una alta implicación por parte del mismo.

En otro estudio llevado a cabo por Schellekens et al. [24] se compararon los resultados obtenidos en la aplicación del programa MBSR en un grupo de pacientes oncológicos con enfermedad avanzada y sus cuidadores principales, con otro programa más psicoeducativo llamado TAU. Se tomaron medidas tanto del distrés psicológico (ansiedad y depresión), como la calidad de vida, y las variables de capacidad mindful, autocompasión y espiritualidad tanto de los pacientes como sus cuidadores principales. Al comparar la aplicación del programa basado en MBSR con el programa psicoeducativo (TAU) observaron que los que habían recibido terapia basada en mindfulness mejoraban en mayor proporción el malestar psicológico y aumentaban el bienestar psicológico 
(mayor autocompasión, más espiritualidad, mayor capacidad de atención plena) que los sujetos del grupo control.

En un estudio llevado a cabo por Thornton et al. [25] se concluye que el programa de MBSR de 8 semanas combinado con el programa "Hope Therapy" (Chaeavens, Feldman, Gum, Michael y Snyder, 2006) ha tenido una aceptación significativa por los pacientes oncológicos. Este estudio concluye unos resultados favorables al incremento de la calidad de vida con una correlación positiva entre el afecto positivo y la medida semanal de la práctica del programa, y una correlación negativa entre ésta y el afecto negativo, observándose una disminución significativa del estrés emocional y la sintomatología ansiosa.

Por otro lado, el meta análisis de Zhang, Wang y Wang [26], realizado mediante una revisión sistemática de siete estudios controlados aleatorizados, con una muestra de 951 pacientes oncológicos y tratados con un programa basado en mindfulness (MBI), concluye también una reducción significativa en ansiedad, depresión y fatiga y un aumento del bienestar autoinformado.

Tras realizar una revisión exhaustiva, se llega a la conclusión de que la aplicación de los programas de mindfulness en oncología contribuyen a un mayor ajuste psicológico en los pacientes oncológicos, una reducción de los niveles de ansiedad y de depresión, mejoría de la calidad del sueño, uso de mejores estrategias de afrontamiento, cambios en el sistema inmune y endocrino, aumento de la calidad de vida y reducción del dolor [27].

En líneas generales, la aplicación de mindfulness presenta un panorama alentador en la intervención terapéutica oncológica. En los últimos años está habiendo un aumento significativo de estudios dónde los datos apuntan a una reducción significativa del distrés psicológico, así como un aumento importante del bienestar y la calidad de vida. Es por ello que los modelos basados en la práctica de mindfulness están en auge, aunque debido a que se trata de técnicas recientes, se requiere la realización de nuevos estudios que confirmen los beneficios observados. Los datos apuntan a un "optimismo precavido" ya que muchos estudios adolecen de los controles metodológicos adecuados.

\section{Programa MBPM de "Respira vida breathworks"}

En la actualidad existen diferentes paquetes terapéuticos de mindfulness aplicados en el ámbito oncológico, concretamente la Terapia Cognitiva Basada en Mindfulness para el cáncer (MBCT- CA) [28], una adaptación de la MBCT (Terapia Cognitiva Basada en Mindfulness para la depresión), y la terapia MBSR en el Centro Tom Baker, que consiste basada en el programa de Kabat-Zinn.

Se trata de un programa psicoeducativo grupal de ocho semanas con estándares de calidad, basado en la evidencia científica y las prácticas contemplativas. Fue creado en 2001 por Vidyamala Burch con el objetivo de poder encontrar una manera efectiva, creativa y compasiva de gestionar el dolor, el estrés y la enfermedad.

El programa MBPM está elaborado a partir del libro "Tú no eres tu dolor" de la editorial Kairos, que ha sido premiado por la British Medical Association, recomendado por el Dr. Jon Kabat Zinn.

El programa se basa en los seis pasos que se van desarrollando progresivamente a lo largo de las semanas de intervención:1) La conciencia es posible; 2) Acercarnos a lo desagradable; 3) Ir en búsqueda de lo agradable; 4) Un recipiente más grande; 5) Humanidad compartida y 6) La elección.

Por otro lado, las prácticas que incluye el programa son: El Recorrido del cuerpo, el ancla de la respiración, los movimientos conscientes, la aceptación compasiva, el tesoro del placer, el corazón abierto, la conexión, los 3MM (Los tres minutos mindfulness) y el programa de esfuerzo equilibrado (Pacing).

Las 8 semanas del programa son:

- Semana 1. El caballo Salvaje.

- Semana 2. Tú no eres tus pensamientos.

- Semana 3. Aprende a responder en lugar de reaccionar.

- Semana 4. Observa cómo se disuelven el sufrimiento y el estrés.

- Semana 5. El placer de las pequeñas cosas.

- Semana 6. La tierna gravedad de la bondad. 
- Semana 7. No estás solo.

- Semana 8. La vida vive a través de ti.

En general, la aplicación del programa MBPM en el ámbito de la salud en pacientes que sufren de dolor, ha contribuido a una reducción del nivel de estrés y catastrofización del dolor, a una reducción significativa de los niveles de depresión y ansiedad, a un incremento en la autoevaluación de la calidad de vida, mejoras significativas en la interferencia del dolor y la severidad, así como en la interferencia de la fatiga en la vida cotidiana. Además ha contribuido a un aumento significativo de la autocompasión, de la atención plena y de la calidad del sueño [28,29,30].

Teniendo en cuenta que la bibliografía revisada proporciona un panorama alentador en la aplicación de mindfulness en el ámbito de la salud, y conociendo que el programa MBPM ha sido diseñado con el objetivo de mejorar la calidad de vida de las personas que sufren estrés, enfermedad y/o dolor, se ha decidido aplicar este programa en pacientes oncológicos para evaluar su eficacia en esta población específica con el objetivo de mejorar el ajuste psicológico general del paciente oncológico. Así como proporcionar una mejoría significativa en la capacidad mindful, autocompasión y bienestar psicológico y una reducción en el malestar psicólogo, del dolor y de las interferencias de este en la vida cotidiana.

\section{Material y métodos}

\subsection{Participantes}

La muestra de pacientes ( $\mathrm{N}=22)$ fue derivada de la unidad de psicooncología de la Asociación Española Contra el Cáncer de Valencia. Se incluyeron a pacientes diagnosticados de cáncer mayores de 18 años tanto en cualquiera de las fases de la enfermedad. Los criterios de exclusión considerados fueron: capacidad cognitiva o trastorno psíquico que impidiese el seguimiento de las instrucciones o incapacidad física.

\subsection{Instrumentos}

Las variables que hemos evaluado en el estudio son:

- Capacidad de atención plena

- Autocompasión

- Bienestar psicológico

- Dolor y su repercusión.

Para evaluar estas variables hemos utilizado los siguientes cuestionarios:

- Cuestionario sociodemográfico ad hoc.

- FFMQ. Escala de Baer [31] adaptada al castellano por Cebolla [32]. Mide la capacidad de atención plena desglosado en cinco Subescalas que son: observación, descripción, capacidad de atención plena, no juzgar, reaccionar con conciencia (sin reactividad).

- SCS. Escala de autocompasión de Neff [33]. Se trata de un cuestionario de 26 ítems que trata de evaluar en qué medida la persona es amable consigo misma. Existen tres factores dentro de la autocompasión: amabilidad, humanidad compartida y capacidad mindful. Estos tres factores se miden en seis escalas que son: mindfulness/ensimismamiento; amabilidad/autocrítica y humanidad compartida/ aislamiento. Cada uno de los ítems es evaluado con una escala Likert que va del 1 al 5, dónde 1 es casi nunca y 5 es casi siempre.

- PANAS. Es un cuestionario bidimensional, que puntúa tanto el afecto positivo como el negativo a través de 20 emociones (10 de afecto negativo y 10 de afecto positivo). Consiste por tanto en 
una estructura bifactorial, con dos dimensiones independientes [34]. Ha sido adaptado para su utilización en España [35].

- $\quad$ SWLS. Esta escala valora en qué medida una persona se siente satisfecha con su vida. Se emplea para medir el bienestar eudaimonico. La SWLS [36] es un instrumento formado por 5 ítems y las respuestas se recogen en una escala tipo Likert de 5 puntos, que oscila desde muy en desacuerdo (1) hasta muy de acuerdo. Está adaptada al castellano por [37].

- SVS. Evalúa la percepción subjetiva de vitalidad de una persona. Se emplea para medir el bienestar eudaimonico. Ryan y Frederick [38] desarrollaron una escala de vitalidad subjetiva (The Subjective Vitality Scale - SVS -) para valorar los sentimientos subjetivos de viveza y energía globalmente. Se empleará la SVS validada al castellano [39]. Esta versión está compuesta por 6 ítems. Los resultados se recogen en una escala tipo Likert de 5 puntos, que oscila desde no es verdad (1) hasta verdadero (5).

- CBD. Es la versión española del cuestionario Brief Pain Inventory (BPI) para medir la intensidad del dolor de causa neoplásica y su impacto en las actividades de la vida diaria en pacientes con cáncer [40].

- Indicadores ad hoc. Se trata de un material elaborado ad hoc para evaluar los indicadores que se han tratado de mejorar con la intervención grupal basada en mindfulness. Es un cuestionario que trata de recoger los niveles de aceptación, serenidad, insomnio, dolor, ansiedad, irritabilidad, tristeza, regulación emocional, autocompasión y rumiación de la muestra valorados de 0 a 10 .

\subsection{Procedimiento}

Todos los pacientes fueron derivados de la Unidad de psicooncología de AECC Valencia, y tras ser informados fueron entrevistados y evaluados antes de la intervención y después de la misma. Toda la intervención y recogida de muestra se realizó siguiendo las normas éticas de investigación en humanos. Se mantuvieron la confidencialidad y el anonimato mediante la codificación de los datos.

La intervención psicológica del programa se ha realizado durante un periodo de dos meses, con 8 sesiones de frecuencia semanal y de 2,5 horas de duración (incluyendo descanso). Tras el mes de finalización de la sesión 8 se hizo una sesión de seguimiento. Mediante el programa MBPM creado por Vidyamala Burch se enseña una forma más sana de relacionarse con el sufrimiento, mediante la autogestión.

El material empleado en las sesiones ha sido el siguiente:

- Esterillas

- Mantas

- Cojines de meditación

- Cuenco tibetano

- Manual de tareas.

- Meditaciones grabadas con link de acceso.

Cada una de las sesiones ha constado de diferentes partes diferenciadas: revisión de tareas con rueda experiencial de los integrantes del grupo, práctica de meditación formal, psicoeducativo del tema de esa semana y pauta de tareas para casa, con una duración aproximada de $2.5 \mathrm{~h}$.

\subsection{Análisis estadísticos}

Para todas las variables recogidas se realizaron los estadísticos descriptivos y frecuencias más comunes. En el caso de las variables nominales se presentaron los porcentajes (\%) de cada una de las categorías previamente definidas. Para las variables discretas se determinaron la media y la SEM (media del error estándar). Para la comparación de las puntuaciones obtenidas en los cuestionarios antes (pre) y después de la terapia (post) se utilizó la prueba estadística t de Student para muestras 
relacionadas. El nivel de significación estadístico considerado fue del 5\% y el paquete estadístico utilizado para el análisis fue el Statistical Package for the Social Sciences (SPSS) versión 25.0.

\section{Resultados}

De las 22 que iniciaron el programa, un 91\% lo finalizaron. Hubo muy buena adhesión al programa, la media de asistencia fue del $75 \%$, y nadie faltó a más del $80 \%$ de las sesiones.

La media de edad de los participantes fue de 52,68 \pm 1,81 años. En cuanto al perfil de la muestra, se observa que el $81.8 \%$ eran mujeres, al dividir la muestra en función del estado civil de los participantes el $50 \%$ estaban casados, frente al $18.2 \%$ de solteros y el $31.8 \%$ de separados/divorciados. Mayoritariamente tenían estudios medios (50\%) y estaban jubilados ( $40.9 \%$ ) o de baja (31.9\%).

Los participantes llevaban, en su mayoría, más de 12 meses diagnosticados $(72,7 \%)$ y principalmente de cáncer de mama (54.5\%). Otros participantes tenían diagnósticos de linfoma de Hodking, renal, óseo y sarcoma. El 63.6\% de la muestra estaba libre de enfermedad, el $9.1 \%$ estaba con tratamiento quimioterápico, el $18.2 \%$ estaba recibiendo otros tratamientos tanto curativos como paliativos, en contraposición de $4.5 \%$ que no estaba recibiendo tratamiento.

En el periodo de realización del programa MPBM el 72.7\% de la muestra no estaba recibiendo ayuda psicológica, pero el $63,6 \%$ estaba tomando psicofármacos. Además, el $72.7 \%$ de los participantes referían sucesos vitales estresantes (SVE).

La puntuación de todas las escalas mejoró a la finalización del programa respecto a los valores basales (pre).

\subsection{Capacidad de atención plena}

Como se puede observar en la figura 1, la capacidad general de atención plena de los participantes evaluada con el cuestionario FFMQ aumentó significativamente tras la terapia (109.95 \pm 3.88 vs. $138.75 \pm 3.95)(\mathrm{p}<0.05)$. Asimismo, se obtuvieron mejoras significativas en las puntuaciones de las cinco dimensiones del cuestionario (observación (24.27 \pm 1.44 vs. $31.15 \pm 1.26$ ), descripción ( $24.09 \pm 1.19$ vs. $28.85 \pm 1.11)$, atención plena ( $21.91 \pm 1.07$ vs. $27.55 \pm 1.23)$, no juzgar (19.56 \pm 1.13 vs. $27.20 \pm 0.76)$ y no reactividad $(19.59 \pm 1.13$ vs. $24.90 \pm 0.76))$ tras la aplicación del programa $(\mathrm{p}<0.05)$.

\section{Capacidad de atención plena}

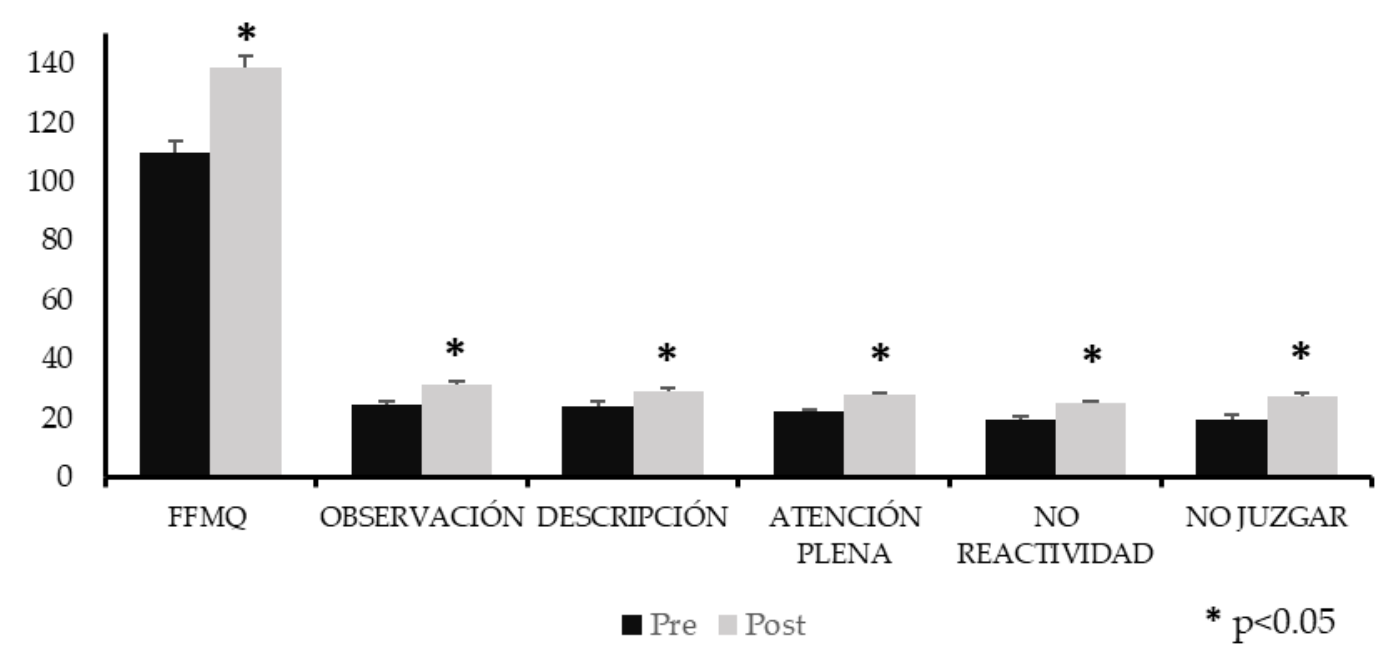

Figura 1. Evaluación de la capacidad de atención plena (PRE-POST terapia). Esta capacidad ha sido valorada con el FFMQ mediante la categoría general, así como con las cinco subescalas: OBS (Observación); DESC (Descripción); AP (Atención plena); NJ (No Juzgar) y NR (No reactividad). 


\subsection{Capacidad de autocompasión}

Para valorar en qué medida la persona es amable consigo misma se utilizó el cuestionario de autocompasión de Neff. Los resultados, tras el análisis de prueba t para muestras relacionadas realizado (figura 2), reportaron un aumento significativo de dicha capacidad en los participantes del estudio $(69.91 \pm 3.36$ vs. $91.80 \pm 4.18)(\mathrm{p}<0.05)$. Además, los sujetos mostraron mayores niveles de amabilidad ( $26.59 \pm 1.40$ vs. $34.00 \pm 1.46)$, humanidad compartida ( $22.91 \pm 1.27$ vs. $30.25 \pm 1.33)$ y capacidad mindful $(20.41 \pm 0.99$ vs. $28.45 \pm 1.22)$ tras finalizar la terapia, evaluadas mediante el cuestionario SCS.

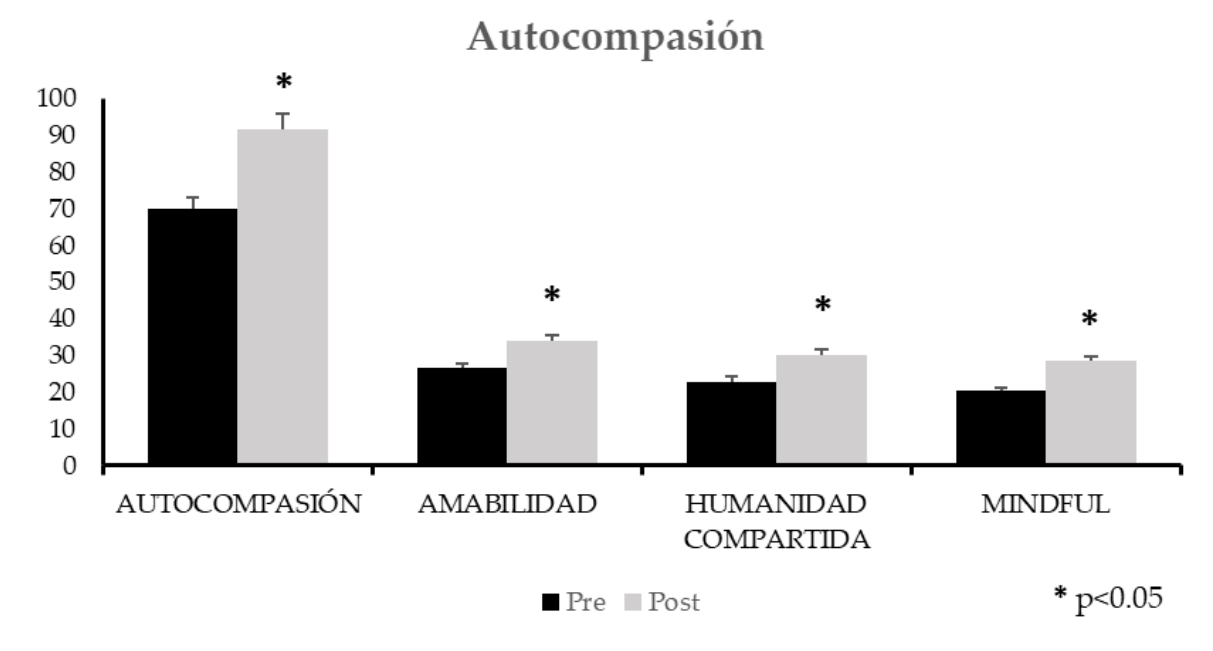

Figura 2. Evaluación de la capacidad de autocompasión (PRE-POST terapia). Esta capacidad ha sido obtenida con la medida general del cuestionario de autocompasión SCS y las escalas de amabilidad, humanidad compartida y capacidad mindful.

\subsection{Bienestar psicológico}

El bienestar psicológico es otra variable que influye en la eficacia del programa. Los sujetos mostraron un aumento significativo en las puntuaciones tras la terapia de las escalas de satisfacción con la vida ( $13.73 \pm 0.73$ vs. $16.50 \pm 0.72)$, vitalidad subjetiva $(16.50 \pm 0.74$ vs. $17.27 \pm 0.80)(p<0.05)$ y afecto positivo del cuestionario Panas $(27.18 \pm 0.73$ vs. $32.10 \pm 0.72)(\mathrm{p}<0.05)$ (figura 4). Además, los sujetos presentaron puntuaciones más bajas en la escala de Afecto Negativo del cuestionario Panas al finalizar la terapia $(21.45 \pm 1.50)$ que al inicio de la misma $(32.32 \pm 1.93)(\mathrm{p}<0.05)$.

\section{Bienestar psicológico}

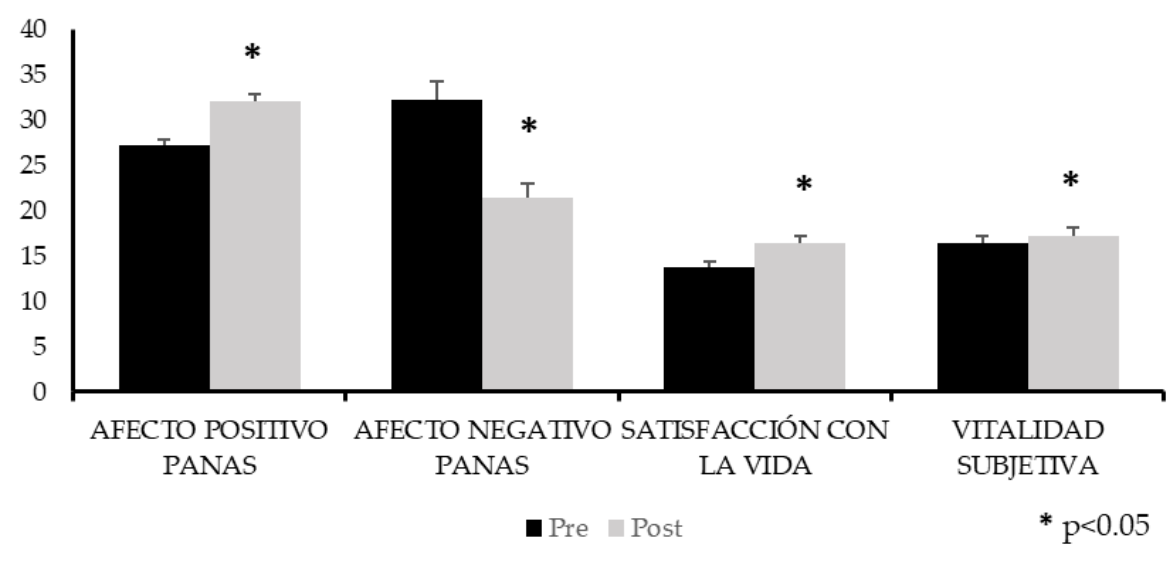

Figura 3. Evaluación del bienestar psicológico (PRE-POST terapia). Esta capacidad ha sido valorada mediante el cuestionario Panas, escala de satisfacción con la vida (SWLS) y vitalidad subjetiva (SVS). 


\subsection{Niveles de dolor}

Para valorar el nivel de dolor que estaban sufriendo los participantes del estudio se utilizó la escala CBD. Los resultados mostraron una disminución significativa en la intensidad de dolor medio ( $3.27 \pm 0.72$ vs. $2.15 \pm 0.46)$ y actual $(2.95 \pm 0.71$ vs. $1.80 \pm 0.46)(\mathrm{p}<0.05)$. Los niveles de dolor máximo y mínimo también se redujeron, pero estas diferencias no fueron significativas. Por otro lado, también se produjeron una reducción en las interferencias del dolor en la actividad general (3.91 \pm 0.79 vs. 2.10 $\pm 0.57)$, estado de ánimo ( $3,86 \pm 0.77$ vs. $2.05 \pm 0.51)$ capacidad de caminar ( $3,36 \pm 0.82$ vs. $2.25 \pm 0.74)$, relaciones ( $3.68 \pm 0.80$ vs. $1.75 \pm 0.53)$, sueño $(4.22 \pm 0.83$ vs. $2.80 \pm 0.77)$ y disfrute de la vida ( $3.68 \pm$ 0.74 vs. $2.40 \pm 0.63)$ tras la finalización del programa $(\mathrm{p}<0.05)$. Por otro lado, aunque las interferencias en el trabajo también se redujeron, no fueron significativas.

\section{Dolor}

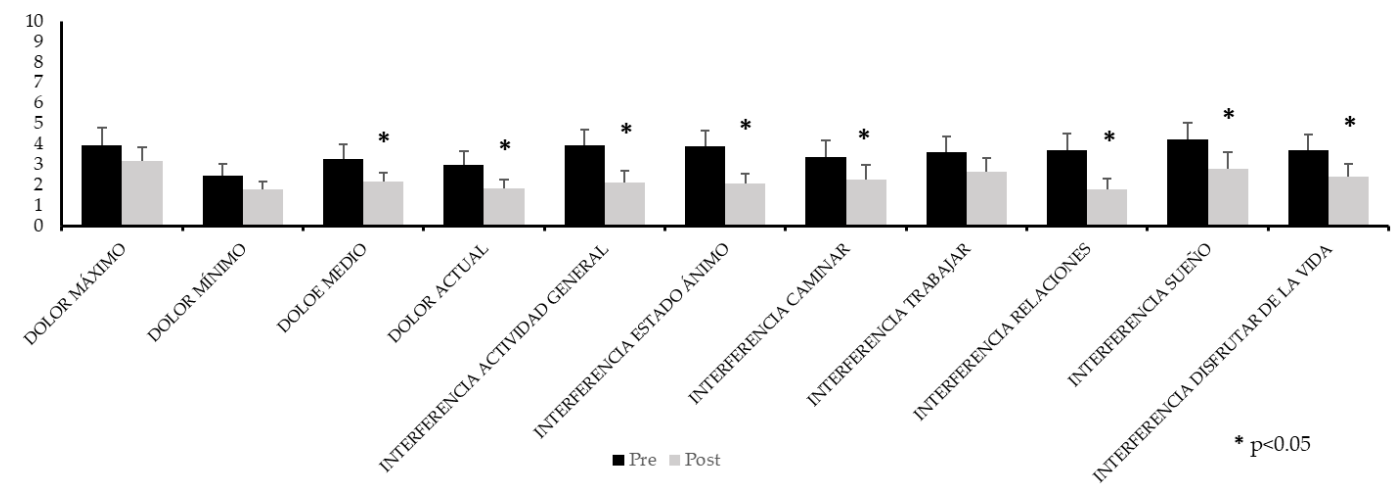

Figura 4. Evaluación de la capacidad de autocompasión (PRE-POST terapia). Esta capacidad ha sido obtenida con el cuestionario de autocompasión de Neff (2013) y CSC.

\subsection{Indicadores de bienestar ad hoc}

El análisis comparativo de los resultados obtenidos a partir de los indicadores de bienestar psicológico ad hoc indica que existen diferencias significativas en todas las variables. Los sujetos mostraron una puntuación significativamente mayor tras finalizar la terapia en las variables aceptación ( $4.72 \pm 0.36$ vs. $8.15 \pm 0.32)$, serenidad (7.70 \pm 0.33 vs. $6.36 \pm 0.33)$, regulación emocional $(4.77 \pm 0.44$ vs. $7.15 \pm 0.37)$ y autocompasión $(3.81 \pm 0.36$ vs. $7.35 \pm 0.50)(\mathrm{p}<0.05)$. Así como disminución en las puntuaciones de insomnio ( $6.36 \pm 0.54$ vs. $4.80 \pm 0.61)$, dolor $(4.86 \pm 0.75$ vs. $3.00 \pm 0.48)$, ansiedad (6.82 \pm 0.47 vs. $4.25 \pm 0.53)$, irritabilidad ( $6.68 \pm 0.57$ vs. $3.50 \pm 0.44)$, tristeza $(7.00 \pm 0.49$ vs. $3.10 \pm 0.38)$ y rumiación $(7.64 \pm 0.44$ vs. $4.30 \pm 0.50)(\mathrm{p}<0.05)$ (figura 5$)$.

\section{Indicadores Bienestar ad hoc}

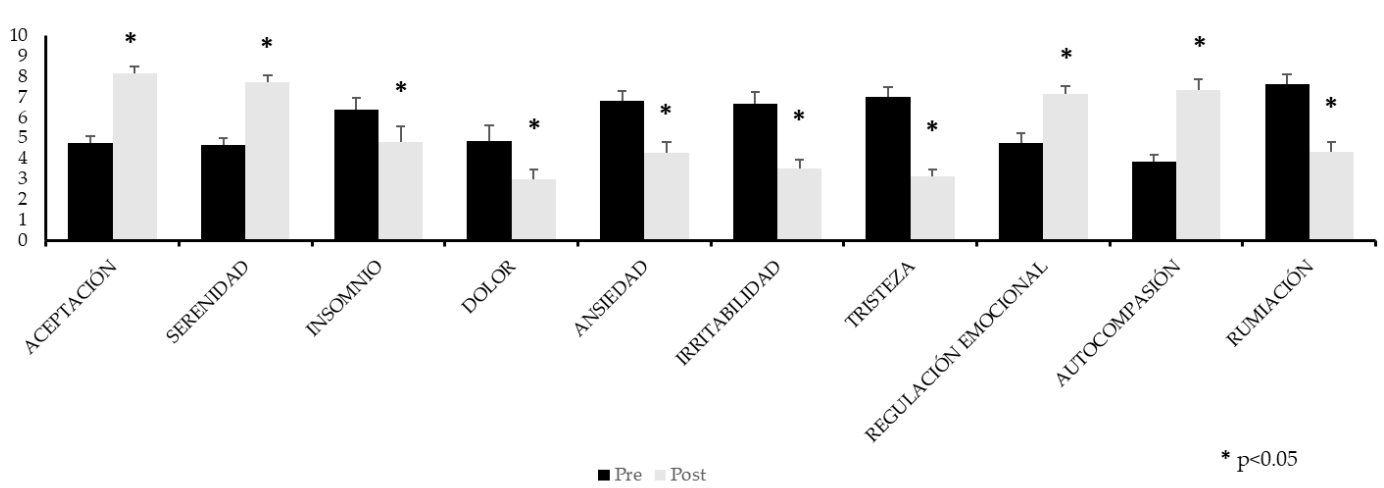

Figura 5. Evaluación de indicadores ad hoc relacionados con la intervención grupal (PRE-POST terapia). 


\section{Discusión}

En líneas generales los resultados mostraron la existencia un aumento significativo en la capacidad de atención plena; un aumento significativo de autocompasión, amabilidad y humanidad compartida; una mejoría significativa en el bienestar psicológico, aumentando el afecto positivo, satisfacción con la vida, vitalidad subjetiva y disminuyendo el afecto negativo; una disminución en el dolor estadísticamente significativa, así como menores interferencias del dolor en las actividades. Este cambio positivo ha sido encontrado también en estudios previos [41,42].

Los resultados obtenidos en el presente estudio muestran que, tras la intervención, los sujetos mejoran en su capacidad de atención plena. Estos datos concuerdan con estudios previos $[43,44]$ Además, a nivel específico, se encuentran mejorías en las capacidades de observación, descripción, atención plena, no juzgar y no reactividad. Otros estudios, en esta línea, relatan que este tipo de terapias tienen éxito ya que favorece actitudes de aproximación frente a evitación $[15,16]$ y permiten una visión más clara de las experiencias, sin pensamientos que distorsionan la realidad [18]. Estos incrementos en la atención plena favorecen, además, al aumento del bienestar psicológico, autocompasión y reducción de rumiaciones y estrés percibido [43].

La autocompasión ha sido evaluada antes y después de implementar el programa MPBM. Los datos muestran que ha aumentado el nivel de esta capacidad en el grupo al finalizar el tratamiento. Además, específicamente, se ha observado un aumento de amabilidad, humanidad compartida y capacidad mindful. Otras investigaciones muestran la relevancia que tiene esta variable en la elaboración de situaciones complicadas como puede ser la enfermedad oncológica [33,45]. Estudios previos muestran mayores niveles de esta variable en personas que utilizan la meditación frente a los que no practican [24,29,30,46,47]. A su vez, esta variable repercute en el bienestar de la persona [47].

Con respecto a ese bienestar, en la presente muestra se observa un aumento del bienestar psicológico evaluado a través de una mayor satisfacción con la vida, vitalidad subjetiva y afecto positivo, así como una reducción del afecto negativo. Estudios previos que evalúan la eficacia de intervenciones basadas en mindfulness muestran mejoras significativas en el malestar emocional tras recibir la terapia $[17,23,44]$. En población oncológica los estudios son más reducidos, pero también se obtienen resultados prometedores al respecto, se observa un aumento significativo el bienestar emocional de pacientes y familiares frente al grupo control tras finalizar el programa [24]. Otro estudio, encontró mayores niveles de capacidad mindful que estaban relacionados con una reducción de los niveles de distrés y un aumento de los niveles de vitalidad, afecto positivo y satisfacción con la vida [48].

Por otro lado, el dolor es una variable presente tanto en el diagnóstico como en el tratamiento del cáncer. Los resultados de la terapia muestran una reducción de la intensidad de dolor, así como una disminución en las interferencias de este en las actividades de la vida cotidiana como el estado de ánimo, la capacidad para caminar, las relaciones sociales, el sueño o el disfrute de la vida. En otros estudios también ha sido ampliamente demostrado que los programas de mindfulness bien estructurados pueden ayudar a la disminución del estrés y el malestar psicológico $[17,23,44]$, pero estudios como el de Speca et al., [20] muestran que también ayuda a paliar el dolor físico. En su estudio presentan que la eficacia de un programa basado en mindfulness se puede comparar con la eficacia obtenida a través del tratamiento con analgésicos y morfina. La práctica continuada de esta técnica ayuda a amortiguar y manejar mejor el sufrimiento asociado a la enfermedad. Otros estudios más recientes, también apoyan estos resultados [21,46].

Por último, al elaborar el protocolo del programa MPBM se valoró la necesidad de evaluar dimensiones importantes que se trabajan durante las sesiones. Por ello, se elaboraron una serie de indicadores valorados de 0 a 10. Los datos muestran que tras la intervención mejora la aceptación, serenidad, regulación emocional, autocompasión. Por otro lado, disminuyen los problemas de sueño, la irritabilidad, ansiedad, tristeza y la rumiación. En estudios previos se puede observar como también se obtienen mejorarías en estas variables, que a su vez aumenta la calidad de vida de las personas afectadas [29,30,46,48]. En cuanto a la regulación emocional, estos resultados avalan los encontrados en estudios previos [23,49]. León et al. [23] encontraron una disminución significativa de valores de diagnóstico de depresión en el grupo experimental, frente a una ausencia de variación 
en el grupo control. En el caso de la ansiedad, se reduce en ambos grupos, siendo más acusada en el grupo que ha sido tratado con un programa de mindfulness.

\section{Conclusiones}

Tal y como muestran los resultados, el programa MBPM DE RESPIRA VIDA BREATWORKS en pacientes oncológicos mejora el ajuste psicológico de los participantes. También se puede observar como mejoran las variables específicas de la práctica de mindfulness como la atención plena, la autocompasión, la capacidad mindful, la serenidad, aceptación, etc. Cabe destacar la importancia de replicar este estudio con un grupo control y un $\mathrm{N}$ mayor para poder generalizar estos resultados, aunque estos son prometedores. Estos resultados ayudan en la búsqueda de estrategias que mejoraran los efectos deletéreos que la enfermedad oncológica puede provocar en las personas que la sufren.

Agradecimientos: Esta investigación ha sido posible gracias a la ayuda proporcionada por la Junta Asociada Provincial de Valencia de la asociación Española Contra el Cáncer y a RespiraVida/Breathworks.

Contribución de los autores: Alonso-Llácer ha implementado el programa y ha recogido los datos para su posterior estudio. Ramos-Campos ha analizado e interpretado los datos del estudio. Alonso-Llácer y RamosCampos han realizado la redacción del presente artículo y han aprobado su versión final..

Conflictos de Intereses: los autores no declaran conflicto de intereses.

\section{Referencias Bibliográficas}

1. Martin MJ, Matallanes MB, Pérez J. El impacto psicológico del cáncer de pulmón en el paciente y su familia. Mapfre Medicina 2007;18(2):108-113.

2. Díaz Faciolince VE, Ruiz M, Flórez C, Urrea Cosme YS, Córdoba Sánchez V, Arbeláez Álvarez CC, et al. Impacto familiar del diagnóstico de muerte inminente. 2013.

3. Font A, Rodríguez E. Eficacia de las intervenciones psicológicas en mujeres con cáncer de mama. Psicooncología 2007;4(2/3):423.

4. Jassim GA, Whitford DL, Hickey A, Carter B. Psychological interventions for women with nonmetastatic breast cancer. 2015.

5. Cruzado JA. Tratamiento psicológico en pacientes con cáncer. Síntesis; 2010.

6. Gaviria AM, Vinaccia S, Riveros MF, Quiceno JM. Calidad de vida relacionada con la salud, afrontamiento del estrés y emociones negativas en pacientes con cáncer en tratamiento quimioterapéutico. Psicología desde el Caribe 2011:50-75.

7. Cruzado JAC, Araña Z, Hernández M. Problemas psicológicos en pacientes con cáncer: dificultades de detección y derivación al psicooncólogo. Psicooncología: investigación y clínica biopsicosocial en oncología 2007;4(1):179-192.

8. Mera PC, Ortiz M. La relación del optimismo y las estrategias de afrontamiento con la calidad de vida de mujeres con cáncer de mama. Terapia psicológica 2012;30(3):69-78.

9. Narváez A, Rubiños C, Gómez R, García A, Cortés-funes F. Valoración de la eficacia de una terapia grupal cognitivoconductual en la imagen corporal, autoestima, sexualidad y malestar emocional (ansiedad y depresión) en pacientes de cáncer de mama. Psicooncología 2008;5(1):93102.

10. Sánchez N, Torres A, Camprubí N, Vidal A, Salamero M. Factores predictores del abandono de la intervención psicológica grupal en una muestra de pacientes con cáncer de mama. Psicooncología 2005;2(1):21-32.

11. Simón V. Mindfulnes y psicología: presente y futuro. Informació psicològica 2013(100):162-170.

12. Kabat-Zinn J. Mindfulness Meditation: Health benefits of an ancient Buddhist practice. Mind/Body Medicine, eds. Goleman D, Gurin J. New York 1993.

13. Thich NH. Transformación y sanación: el sutra de los cuatro fundamentos de la consciencia 1999. 
14. Lutz A, Dunne JD, Davidson RJ. Meditation and the neuroscience of consciousness. Cambridge handbook of consciousness 2007:499-555.

15. Segal ZV, Williams M, Teasdale JD. Mindfulness-based cognitive therapy for depression. : Guilford Publications; 2018.

16. Simón V. Mindfulness y neurobiología. Revista de psicoterapia 2007;66(67):5-30.

17. Brown KW, Ryan RM. The benefits of being present: mindfulness and its role in psychological well-being. J Pers Soc Psychol 2003;84(4):822.

18. Deci EL, Ryan RM. The general causality orientations scale: Self-determination in personality. Journal of research in personality 1985;19(2):109-134.

19. Kabat-Zinn J. Coming to our senses: Healing ourselves and the world through mindfulness. : Hachette UK; 2005.

20. Speca M, Carlson LE, Goodey E, Angen M. A randomized, wait-list controlled clinical trial: the effect of a mindfulness meditation-based stress reduction program on mood and symptoms of stress in cancer outpatients. Psychosom Med 2000;62(5):613-622.

21. Burch V, Penman D. Tú no eres tu dolor: Mindfulness para aliviar el dolor, reducir el estrés y recuperar el bienestar. : Editorial Kairós; 2016.

22. Tacón AM. Mindfulness: existential, loss, and grief factors in women with breast cancer. J Psychosoc Oncol 2011;29(6):643-656.

23. León C, Mirapeix R, Blasco T, Jovell E, Arcusa À, Martín A, et al. Mindfulness para la reducción del malestar emocional en pacientes oncológicos. Estudio comparativo con una intervención psicoeducativa estándar. Psicooncología 2013;10(2-3):263-274.

24. Schellekens MP, van den Hurk, Desiree GM, Prins JB, Molema J, Donders ART, Woertman WH, et al. Study protocol of a randomized controlled trial comparing mindfulness-based stress reduction with treatment as usual in reducing psychological distress in patients with lung cancer and their partners: the MILON study. BMC Cancer 2014;14(1):3.

25. Thornton LM, Cheavens JS, Heitzmann CA, Dorfman CS, Wu SM, Andersen BL. Test of mindfulness and hope components in a psychological intervention for women with cancer recurrence. J Consult Clin Psychol 2014;82(6):1087.

26. Zhang J, Xu R, Wang B, Wang J. Effects of mindfulness-based therapy for patients with breast cancer: A systematic review and meta-analysis. Complement Ther Med 2016;26:1-10.

27. Alonso L, Pintado S, Blasco K, De la Torre O, Muntó E, Diego R, Berenguer M, Julián M, Pérez $\mathrm{M}$, Barreto P. Beneficios del mindfulness en el ajuste psicológico de pacientes oncológicos: una revisión. IX Congreso Nacional de Psicología Clínica en San Sebastián, 2011.

28. Bartley T. Terapia cognitiva basada en mindfulness para el cáncer. : Desclée de Brouwer; 2013.

29. Cusens B, Duggan GB, Thorne K, Burch V. Evaluation of the breathworks mindfulness-based pain management programme: effects on well-being and multiple measures of mindfulness. Clinical Psychology \& Psychotherapy: An International Journal of Theory \& Practice 2010;17(1):63-78.

30. Henriksson J, Wasara E, Rönnlund M. Effects of eight-week-web-based mindfulness training on pain intensity, pain acceptance, and life satisfaction in individuals with chronic pain. Psychol Rep 2016;119(3):586-607.

31. Baer RA, Smith GT, Hopkins J, Krietemeyer J, Toney L. Using self-report assessment methods to explore facets of mindfulness. Assessment 2006;13(1):27-45.

32. Cebolla A, García-Palacios A, Soler J, Guillen V, Baños R, Botella C. Psychometric properties of the Spanish validation of the Five Facets of Mindfulness Questionnaire (FFMQ). The European Journal of Psychiatry 2012;26(2):118-126.

33. Neff KD. The self-compassion scale is a valid and theoretically coherent measure of selfcompassion. Mindfulness 2016;7(1):264-274.

34. Watson D, Clark LA, Tellegen A. Development and validation of brief measures of positive and negative affect: the PANAS scales. J Pers Soc Psychol 1988;54(6):1063. 
35. Sandín B, Chorot P, Lostao L, Joiner TE, Santed MA, Valiente RM. Escalas PANAS de afecto positivo y negativo: validación factorial y convergencia transcultural. Psicothema 1999;11(1):3751.

36. Diener E, Emmons RA, Larsen RJ, Griffin S. The satisfaction with life scale. J Pers Assess 1985;49(1):71-75.

37. Atienza FL, Pons D, Balaguer I, García-Merita M. Propiedades psicométricas de la Escala de Satisfacción con la Vida en adolescentes. Psicothema 2000;12(2):314-319.

38. Ryan RM, Frederick C. On energy, personality, and health: Subjective vitality as a dynamic reflection of well-being. J Pers 1997;65(3):529-565.

39. Balaguer, I, Castillo, I, García-Merita, M, \& Mars, L. Implications of structured extracurricular activities on adolescent's well being and risk behaviors: motivational mechanisms. 9th European Congress of Psychology; 2005.

40. Badia X, Muriel C, Gracia A, Núñez-Olarte JM, Perulero N, Gálvez R, et al. Validación española del cuestionario Brief Pain Inventory en pacientes con dolor de causa neoplásica. Medicina clínica 2003;120(2):52-59.

41. Keng S, Smoski MJ, Robins CJ. Effects of mindfulness on psychological health: A review of empirical studies. Clin Psychol Rev 2011;31(6):1041-1056.

42. Asuero AM, Blanco TR, Pujol-Ribera E, Berenguera A, Queraltó JM. Evaluación de la efectividad de un programa de mindfulness en profesionales de atención primaria. Gaceta Sanitaria 2013;27(6):521-528.

43. Carmody J, Baer RA. Relationships between mindfulness practice and levels of mindfulness, medical and psychological symptoms and well-being in a mindfulness-based stress reduction program. J Behav Med 2008;31(1):23-33.

44. Shapiro SL, Oman D, Thoresen CE, Plante TG, Flinders T. Cultivating mindfulness: effects on well-being. J Clin Psychol 2008;64(7):840-862.

45. Jazaieri H, Jinpa GT, McGonigal K, Rosenberg EL, Finkelstein J, Simon-Thomas E, et al. Enhancing compassion: A randomized controlled trial of a compassion cultivation training program. Journal of Happiness Studies 2013;14(4):1113-1126.

46. Nathan HJ, Poulin P, Wozny D, Taljaard M, Smyth C, Gilron I, et al. Randomized Trial of the Effect of Mindfulness-Based Stress Reduction on Pain-Related Disability, Pain Intensity, HealthRelated Quality of Life, and A1C in Patients With Painful Diabetic Peripheral Neuropathy. Clinical Diabetes 2017;35(5):294-304.

47. Lykins EL, Baer RA. Psychological functioning in a sample of long-term practitioners of mindfulness meditation. Journal of cognitive Psychotherapy 2009;23(3):226-241.

48. Brown CA, Jones AK. Psychobiological correlates of improved mental health in patients with musculoskeletal pain after a mindfulness-based pain management program. Clin J Pain 2013;29(3):233-244.

49. Rushton CH, Sellers DE, Heller KS, Spring B, Dossey BM, Halifax J. Impact of a contemplative end-of-life training program: being with dying. Palliative \& supportive care 2009;7(4):405-414.

(C) 2018 por los autores; Esta obra está sujeta a la licencia de Reconocimiento 4.0 Internacional de Creative Commons. Para ver una copia de esta licencia, visite http://creativecommons.org/licenses/by-nc-nd/4.0/. 